\title{
Systematic review of single-port vs. multi-port surgery for rectal cancer
}

\author{
MITSUYOSHI TEI, TOSHINORI SUEDA, TAE MATSUMURA, HARUNA FURUKAWA, \\ CHIKATO KOGA, MASAKI WAKASUGI, HIROMICHI MIYAGAKI, RYOHEI KAWABATA, \\ MASANORI TSUJIE and JUNICHI HASEGAWA
}

Department of Surgery, Osaka Rosai Hospital, Kita-ku, Sakai, Osaka 591-8025, Japan

Received May 17, 2019; Accepted September 7, 2020

DOI: $10.3892 / \mathrm{mco} .2020 .2186$

\begin{abstract}
The aim of the current systematic review was to compare the short-term clinical and oncological outcomes of single-port surgery (SPS) to multi-port surgery (MPS) for rectal cancer in MEDLINE, PubMed and Cochrane Library from January 2010 to December 2018. A total of 5 clinical controlled studies composed one randomized pilot study and four non-randomized studies with a total of 461 patients were analyzed after a systematic review. A total of 125 patients (27.1\%) underwent SPS and 336 patients (72.9\%) underwent MPS for rectal cancer. The rate of conversion to open surgery was lower in the SPS group compared with the MPS group ( 0.8 vs. $5.4 \%$, respectively). A total of $16.8 \%$ of patients in the SPS group required an additional port to complete the operation. The morbidity rate was lower in the SPS group compared with the MPS group (28.0 vs. $39.0 \%$, respectively). The other short-term clinical outcomes were similar in both groups. The R0 resection rate was $99.0 \%$ in the SPS group and $98.7 \%$ in the MPS group. The oncological clearance was satisfactory and similar in both groups. The current study concluded that SPS can be performed safely and provide satisfactory oncological outcomes in patients with rectal cancer. However, further studies are required to determine the role of SPS in the long-term clinical and oncological outcomes.
\end{abstract}

\section{Introduction}

Single-port surgery (SPS) is a recent advance in minimally invasive techniques. The benefits of SPS included better cosmetic outcomes, less postoperative pain, faster postoperative recovery, and earlier discharge from the hospital, compared with multi-port surgery (MPS) (1-3). Recently, SPS

Correspondence to: Dr Mitsuyoshi Tei, Department of Surgery, Osaka Rosai Hospital, 1179-3 Nagasonecho, Kita-ku, Sakai, Osaka 591-8025, Japan

E-mail: mtei@live.jp

Key words: single-incision laparoscopic surgery, single-port surgery, rectal cancer can provide satisfactory oncological outcomes in patients with colon cancer (4-6).

On the other hand, the usefulness of SPS for rectal cancer is unknown. SPS for rectal cancer is somewhat technically more challenging, and there is no clinical evidence to confirm the safety and feasibility of SPS for rectal cancer. In addition, it is unclear whether SPS is able to ensure the satisfactory oncological clearance in patients with rectal cancer in comparison of MPS. Therefore, in this systematic review, we aimed to compare the feasibility and safety of SPS with those of MPS for rectal cancer in terms of perioperative and short-term oncological outcomes.

\section{Materials and methods}

Literature research. We had systematically collected useful studies from MEDLINE, PubMed and Cochrane Library from 2010 to 2018. The search items were 'Single incision', 'single port', 'single site', 'SILS' and 'rectal cancer'. Manual searches of references from relevant articles were performed when necessary. MPS was defined as laparoscopic surgery using three or more ports by a small incision. SPS was defined as laparoscopic surgery using only one port by a small incision. Articles were selected if the abstract contained data on patients who underwent SPS for rectal cancer. Publications were included if they were randomized controlled trials, case-matched controlled trials, or comparative observational studies, in which patients underwent SPS for rectal cancer. Studies were excluded if they were non-comparative studies, or including surgery involving colon cancer or rectosigmoid cancer. Review articles, conference abstracts, case, letter and other unqualified articles were excluded.

Outcome of interest. We used the following results to compare SPS and MPS for rectal cancer: i) Patient profile including age, sex, body mass index and previous abdominal surgery; ii) operative data including operative time, blood loss, conversion rate and additional port; iii) postoperative outcome including morbidity, mortality, anastomotic leakage, postoperative hospital stay, reoperation and readmission; iv) histopathological findings including tumor size, number of harvested lymph node, length of resected specimen, proximal margin, distal margin and positive resection margin. 


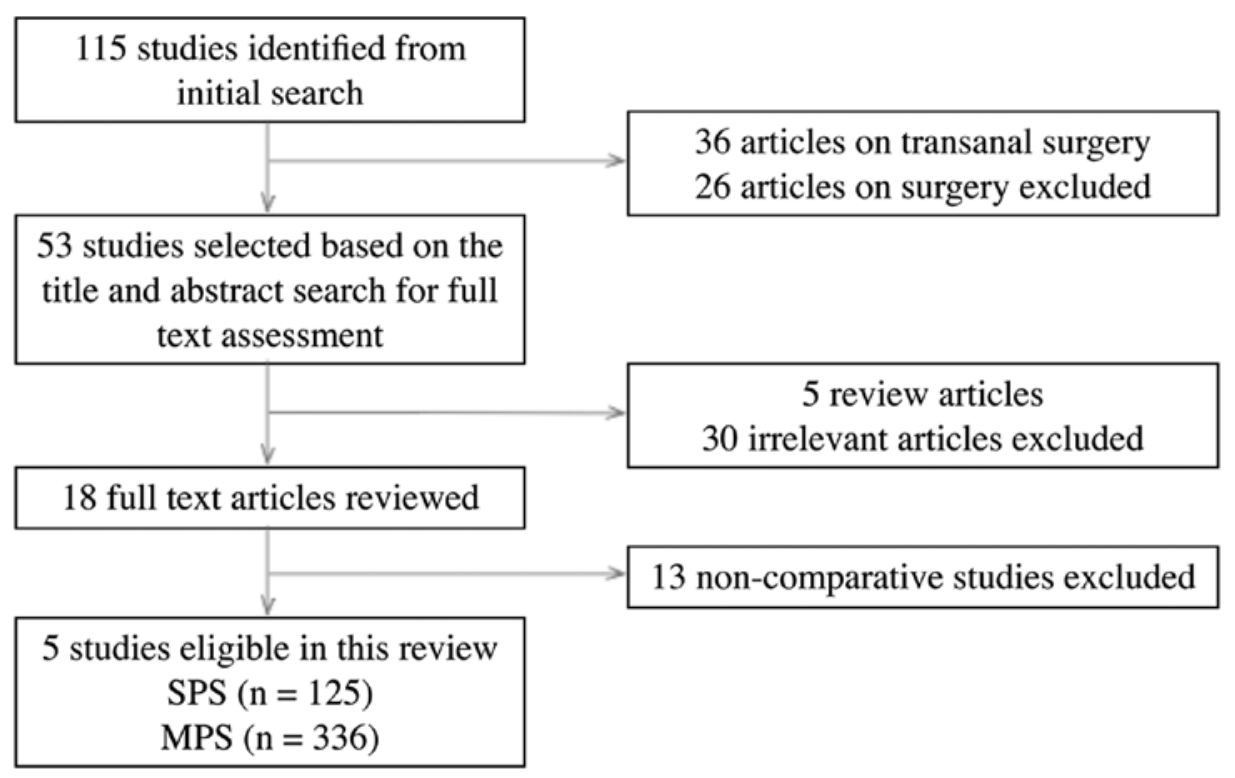

Figure 1. Flowchart of the study search in this systematic review.

Risk of bias evaluation. One randomized controlled trail quality was assessed by the Cochrane Reviewers' Handbook with the Jaded score in three metrics: Randomization, double blindness, and control. Four non-randomized controlled trail qualities were assessed with the Newcastle-Ottawa Scale from three aspects: Patient selection, confirmation of exposure, and comparability of both groups.

\section{Results}

Study characteristics and quality assessment. We found 115 potentially relevant publications by using the above search strategy. Twenty-six publications on robotic-assisted laparoscopic surgery and 36 publications on transanal minimal invasive surgery were excluded. After reviewing titles and abstracts of 53 articles, five review articles, 30 irrelevant articles, and 13 articles on surgical technique were excluded. Finally, five studies were eligible in this review, which included one randomized trial and four comparative studies. All patients who underwent SPS or MPS were confirmed pathologically for rectal cancer (7-11). The flowchart of the selection process for studies included in this review is presented in Fig. 1. We considered that one randomized controlled trail had low quality, and four non-randomized controlled trails had moderate or high quality.

Patient profiles, operative details and postoperative outcomes. Of the patients evaluated by these studies, 125 patients underwent SPS and 336 patients underwent MPS. Table I lists the profiles of the patients from each study, including age, sex, body mass index and previous abdominal surgery.

The operative details show in the Table II. The operative time, blood loss and the conversion rate to open surgery were described in five studies. Levic and Bulu (9) and Nerup et al (10) reported that the operative time was significantly longer in the SPS group than in the MPS group (295 min vs. $248 \mathrm{~min}, \mathrm{P}=0.01$ and $316 \mathrm{~min}$ vs. $269 \mathrm{~min}, \mathrm{P}=0.004$, respectively). Sourrouille et al (11) and Levic and Bulu (9) reported that blood loss was significantly less in the SPS group than in the MPS group (100 $\mathrm{ml}$ vs. $200 \mathrm{ml}, \mathrm{P}=0.01$ and $35 \mathrm{ml} \mathrm{vs}$. $100 \mathrm{ml}, \mathrm{P}=0.006$, respectively). The conversion rate to open surgery was lower in the SPS group than in the MPS group ( 0.8 vs. $5.4 \%$, respectively). $16.8 \%$ of patients in the SPS group required an additional port to allow completion of the operation.

The postoperative outcomes show in Table III. The incidence of morbidity, mortality, anastomotic leakage, postoperative hospital stay and reoperation were reported in all five studies. Morbidity rate was lower in the SPS group than in the MPS group (RR $0.69,95 \%$ CI 0.43 to 1.11 ). Mortality rate was $2.4 \%$ in each group. The incidence of anastomotic leakage was $9.7 \%$ in the SPS group and $10.2 \%$ in the MPS group, respectively (RR $0.79,95 \%$ CI 0.36 to 1.71 ). Postoperative hospital stay was reported as 7 to 12 days in the SPS group and as 7 to 14 days in the MPS group, respectively. Reoperation rate was $8.8 \%$ in the SPS group and $13.4 \%$ in the MP group, respectively (RR $0.95,95 \%$ CI 0.46 to 1.95 ). The incidence of readmission was reported in four studies, $16.2 \%$ in the SPS group and $10.6 \%$ in the MPS group, respectively (RR, 0.1.60; 95\% CI, 0.69 to 3.69). The postoperative pain was reported in two studies. Sourrouille et al (11) reported that the median visual analog scale score on postoperative days 2 was significantly lower in the SPS group than in the MPS group ( 1.5 vs. 4 , respectively, $\mathrm{P}=0.01)$ and the need for dose of morphine was significantly lower in the SPS group than in the MPS group (2.5 vs. $4 \mathrm{mg}$, respectively, $\mathrm{P}=0.02)$. Bulut et al (8) reported that the NRS pain scores were significantly reduced in the SPS group on postoperative days 2, 3 and 4 during both coughing and mobilization. In addition, the patients in the SPS group suffered significantly less pain at rest at $6 \mathrm{~h}$ after surgery and at postoperative days 1, 3 and 4 . All five studies had significant heterogeneity in random effect model by I-square statistics.

Pathological findings and oncological outcomes. The pathological findings show in Table IV. Tumor size and the detail 


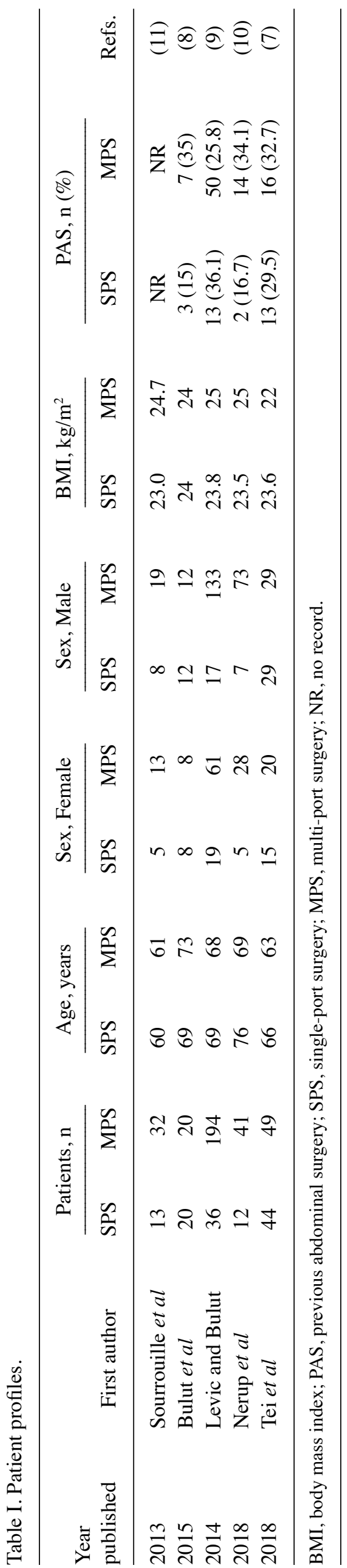

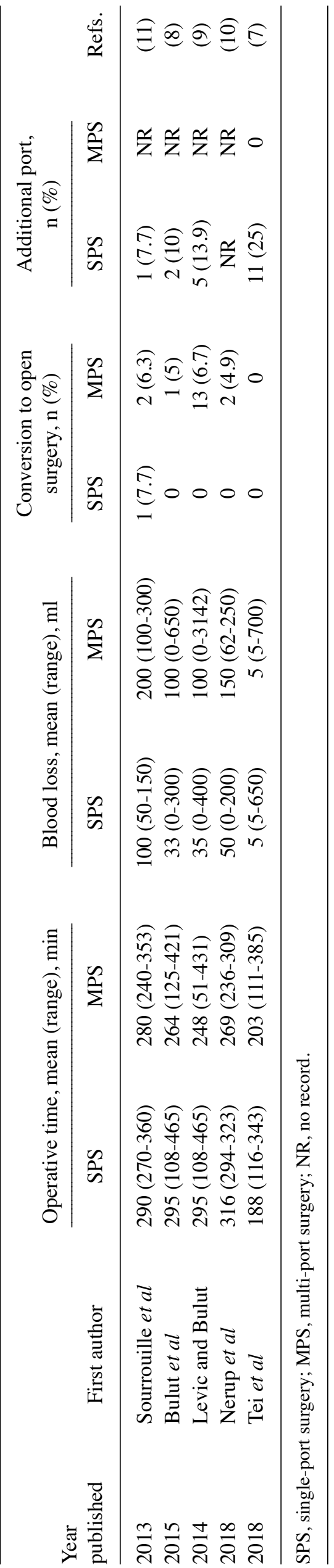




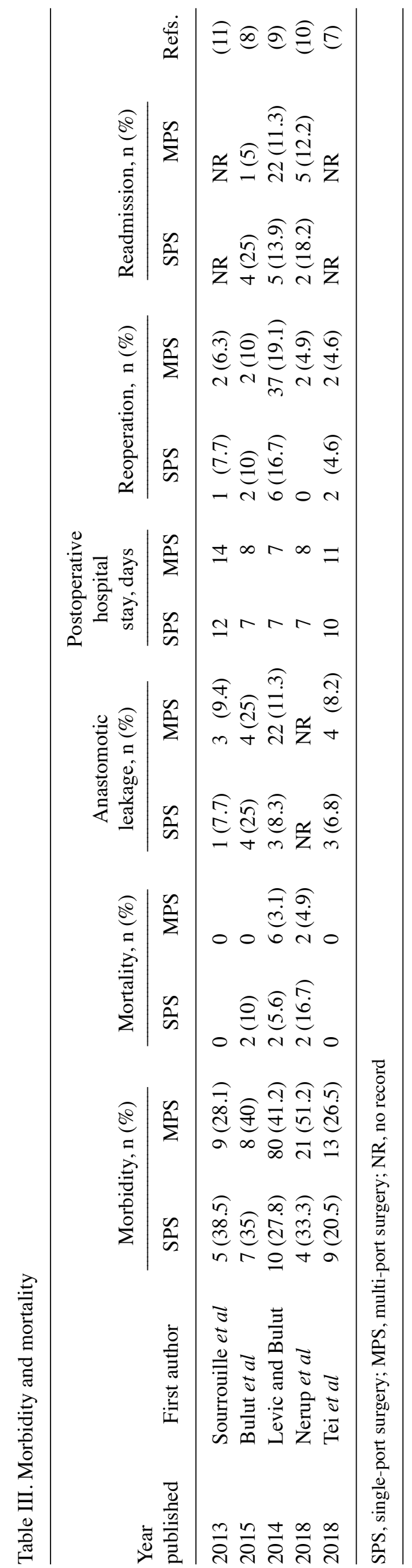

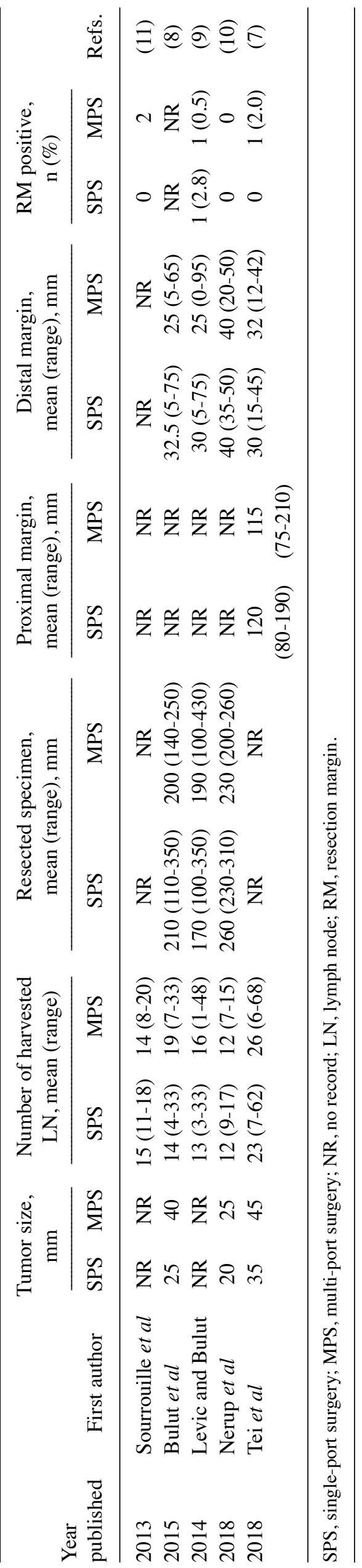


of resected specimen were reported in four studies. The number of harvested lymph node was reported in five studies. Levic and Bulu (9) was reported that the number of harvested lymph node was significantly lower in the SPS group than in the MPS group (13 vs. 16, respectively, $\mathrm{P}=0.047$ ). In the other four studies, there was no significant difference in the number of harvested lymph node. The length of resected specimen, proximal resection margin, and distal margin were similar in both groups. The rate of positive resection margin was $1 \%$ in the SPS group and $1.3 \%$ in the MPS group, respectively.

With regard to survival outcomes, only one study reported 3-year relapse free survival and 5-year overall survival. Tei et al (7) reported that the 3-year relapse free survival and 5-year overall survival rates were 94.7 and $97.4 \%$ in the SPS group, over the median follow-up period of 40 months, respectively. In the MPS group, the 3-year relapse free survival and 5-year overall survival rates were 78.6 and $86.1 \%$, over the median follow-up period of 51 months, respectively.

\section{Discussion}

Recently, surgery for colorectal cancer is shifting to less invasive surgery because of better postoperative short-term surgical results, which include: Lower postoperative morbidity, reduced intraoperative blood loss, less pain, faster recovery and better quality of life $(12,13)$. Moreover, laparoscopic surgery for colorectal cancer is considered to be comparable to long-term results compared with open surgery $(12,13)$.

SPS for colorectal disease was first described in $2008(14,15)$, and has been reported the better short-term surgical outcomes in comparison to MPS since then. However, most of the reports on SPS are concerned with colon cancer (4-6), and whether SPS is better than MPS for rectal cancer still remains unclear.

This systematic review is to compare the clinical outcomes of SPS vs. MPS in patients with rectal cancer. The major findings of this analysis show that SPS for rectal cancer is a safe and feasible approach (as deemed by $16.8 \%$ of patients requiring an additional port and $0.8 \%$ requiring conversion to open surgery), and yields adequate short-term surgical outcomes (e.g., morbidity of $28.0 \%$ and mortality of $2.4 \%$ ) and satisfactory oncological outcomes (e.g., R0 resection rate of $99.0 \%$ ).

With regard to operative factors, two studies reported that operative time was significantly longer in the SPS group and two studies reported that the blood loss is significantly less in the SPS group. Kim et al reported that the learning curve of SPS for sigmoid colon cancer was 61-65 cases according to multidimensional statistical analyses (16). Li et al reported that the experienced MPS surgeons achieved the technical competence after the 44th case of SPS plus one port in patients with sigmoid colon cancer and upper rectal cancer (17). SPS for rectal cancer is technically more challenging and require more cases for proficiency, and we consider this result is likely little of statistical significance.

The conversion rate to open surgery was significantly lower in the SPS group than in the MPS group (0.8 vs. 5.4\%, $\mathrm{P}=0.032$ ). However, $16.8 \%$ in the SPS group had required an additional port to complete the operation, due to fixation of tumor, severe pelvic fibrosis or distal rectum division. In a previous study, the conversion rate of SPS for rectal cancer was $8 \%$ (18). Hirano et al reported that SPS plus one port was safe and feasible for rectal cancer to overcome the technical difficulties, including mobilization and rectum division (19). We suggest that it is most reasonable to insert an additional port at the time when SPS is judged technically difficult.

With regard to postoperative outcomes, the morbidity and mortality rate in the SPS group were 28.0 and $2.4 \%$, respectively, and comparable to the short-term outcomes of conventional laparoscopic surgery for rectal cancer in a recent randomized control trial (20). Anastomotic leakage, reoperation, postoperative hospital stay and readmission were similar in both groups, and also comparable to the above trial.

Reduction of postoperative pain is very important for early recovery, and it is well known that the transition from open surgery to laparoscopic surgery is associated with reduction of postoperative pain. Sourrouille et al (11) and Bulut et al (8) revealed reduction of postoperative pain scores following SPS compared to MPS in their studies. Although it is controversial whether SPS contributes to the reduction of postoperative pain compared with MPS at present, this innovative surgical technique may be involved in postoperative pain reduction.

The maintenance of the surgical oncological outcome is the most important factor in the treatment of rectal cancer. The oncological outcomes, including number of harvested lymph nodes, length of resected specimen, proximal dissection margin, distal dissection margin, and residual tumor status did not differ between groups. In particular, residual tumor status was negative in $99 \%$ cases of the SPS group, and comparable to the recent randomized controlled trials $(20,21)$. With regard to long-term oncological outcomes, Tei et al reported that the 3-year relapse-free survival rate and 3-year overall survival rate in the SPS group were 94.7 and $97.4 \%$, over the median follow-up period of 40 months, respectively (7). The other four studies did not report for the long-term outcomes. The COLOR II Study Group reported that the 3-year relapse-free survival rate and 3 -year overall survival rate in the laparoscopic surgery group were 74.8 and $86.7 \%$, respectively (22). Jeong et al reported that the 3-year relapse-free survival rate and 3-year overall survival rate in the laparoscopic surgery group were 79.2 and $91.7 \%$, respectively (23). The long-term outcomes of SPS for rectal cancer should be needed for further research.

This systematic review being compared the clinical outcomes between the SPS and MPS for rectal cancer has several important limitations. First, five studies composed of one randomized controlled study and four non-randomized studies with small number of patients, which were not the highest quality of evidence, were a limitation that might be affect the outcomes and induce selection bias, although the majority of the assessed outcomes across all papers had no dramatic conflicts in the findings. Second, there is a difference in terms of preoperative assessment, including tumor location (upper rectum, lower rectum, or distance from anal verge), clinical TNM classification and preoperative treatment. Third, there is a possibility that the results may be influenced by various surgical techniques and devices in the SPS group. Fourth, the long-term oncological outcome is controversial because of insufficient follow-up period after surgery.

This study confirmed the safety and feasibility of SPS for rectal cancer, with slighter postoperative pain, lower conversion rate to open surgery, lower postoperative complication 
rate and satisfactory oncological clearance. In the future, more randomized controlled trials with a large number of cases are needed to demonstrate the clinical and prognostic impact of the SPS for rectal cancer.

\section{Acknowledgements}

Not applicable.

\section{Funding}

No funding was received.

\section{Availability of data and materials}

All the datasets generated and analyzed during the present study are available from the corresponding author on reasonable request.

\section{Authors' contributions}

MTe designed and wrote the systematic review for the topic highlight. TM, HF, CK, MW, HM, RK, and MTs collected the literature. MTe and TS analyzed and interpreted the data and provided the clinical suggestion. TS, TM, HF, CK, MW, HM, RK, and MTs and JH critically revised the article. All authors read and approved the final version of the manuscript.

\section{Ethics approval and consent to participate}

Not applicable.

\section{Patient consent for publication}

Not applicable.

\section{Competing interests}

The authors declare that the have no competing interests.

\section{References}

1. Papaconstantinou HT and Thomas JS: Single-incision laparoscopic colectomy for cancer: Assessment of oncologic resection and short-term outcomes in a case-matched comparison with standard laparoscopy. Surgery 150: 820-827, 2011.

2. Champagne BJ, Papaconstantinou HT, Parmar SS, Nagle DA, Young-Fadok TM, Lee EC and Delaney CP: Single-incision versus standard multiport laparoscopic colectomy: A multicenter, case-controlled comparison. Ann Surg 255: 66-69, 2012.

3. Poon JT, Cheung CW, Fan JK, Lo OS and Law WL: Single-incision versus conventional laparoscopic colectomy for colonic neoplasm: A randomized, controlled trial. Surg Endosc 26: 2729-2734, 2012.

4. Takemasa I, Uemura M, Nishimura J, Mizushima T, Yamamoto H, Ikeda M, Sekimoto M, Doki Y and Mori M: Feasibility of single-site laparoscopic colectomy with complete mesocolic excision for colon cancer: A prospective case-control comparison. Surg Endosc 28: 1110-1118, 2014.

5. Katsuno G, Fukunaga M, Nagakari K, Yoshikawa S, Azuma D and Kohama S: Short-term and long-term outcomes of single-incision versus multi-incision laparoscopic resection for colorectal cancer: A propensity-score-matched analysis of 214 cases. Surg Endosc 30: 1317-1325, 2016.
6. Tei M, Wakasugi M and Akamatsu H: Comparison of perioperative and short-term oncological outcomes after single- or multiport surgery for colorectal cancer. Colorectal Dis 17: O141-O147, 2015.

7. Tei M, Otsuka M, Suzuki Y, Kishi K, Tanemura M and Akamatsu H: Safety and feasibility of single-port laparoscopic low anterior resection for upper rectal cancer. Am J Surg 216: 1101-1106, 2018.

8. Bulut O, Aslak KK, Levic K, Nielsen CB, Rømer E, Sørensen S, Christensen IJ and Nielsen HJ: A randomized pilot study on single-port versus conventional laparoscopic rectal surgery: Effects on postoperative pain and the stress response to surgery. Tech Coloproctol 19: 11-22, 2015.

9. Levic K and Bulut O: The short-term outcomes of conventional and single-port laparoscopic surgery for rectal cancer: A comparative non-randomized study. Minim Invasive Ther Allied Technol 23: 214-222, 2014.

10. Nerup N, Rosenstock S and Bulut O: Comparison of single-port and conventional laparoscopic abdominoperineal resection. J Minim Access Surg 14: 27-32, 2018.

11. Sourrouille I, Dumont F, Goéré D, Honoré C and Elias D: Resection of rectal cancer via an abdominal single-port access: Short-term results and comparison with standard laparoscopy. Dis Colon Rectum 56: 1203-1210, 2013.

12. Lacy AM, Garcia-Valdecasas JC, Delgado S, Castells A, Taurá P, Piqué JM and Visa J: Laparoscopy-assisted colectomy versus open colectomy for treatment of non-metastatic colon cancer: A randomised trial. Lancet 359: 2224-2229, 2002.

13. Leung KL, Kwok SP, Lam SC, Lee JF, Yiu RY, Ng SS, Lai PB and Lau WY: Laparoscopic resection of rectosigmoid carcinoma: Prospective randomised trial. Lancet 363: 1187-1192, 2004.

14. Remzi FH, Kirat HT, Kaouk JH and Geisler DP: Single-port laparoscopy in colorectal surgery. Colorectal Dis 10: 823-826, 2008.

15. Bucher P, Pugin F and Morel P: Single port access laparoscopic right hemicolectomy. Int J Colorectal Dis 23: 1013-1016, 2008.

16. Kim CW, Kim WR, Kim HY, Kang J, Hur H, Min BS, Baik SH, Lee KY and Kim NK: Learning curve for single-incision laparoscopic anterior resection for sigmoid colon cancer. J Am Coll Surg 221: 397-403, 2015

17. Li J, Wang Y, Liu D, Zhou H, Mou T, Li G and Deng H: Multidimensional analyses of the learning curve for single-incision plus one port laparoscopic surgery for sigmoid colon and upper rectal cancer. J Surg Oncol 117: 1386-1393, 2018.

18. Gash K, Bicsak M and Dixon A: Single-incision laparoscopic surgery for rectal cancer: Early results and medium-term oncological outcome. Colorectal Dis 17: 1071-1078, 2015.

19. Hirano Y, Hattori M, Douden K, Shimada M and Hashizume Y: Short-term clinical and oncological outcomes after single-incision plus one port laparoscopic anterior resection for rectal cancer. Dig Surg 35: 111-115, 2018.

20. van der Pas MH, Haglind E, Cuesta MA, Fürst A, Lacy AM, Hop WC and Bonjer HJ; COlorectal cancer Laparoscopic or Open Resection II (COLOR II) Study Group: Laparoscopic versus open surgery for rectal cancer (COLOR II): Short-term outcomes of a randomised, phase 3 trial. Lancet Oncol 14: 210-218, 2013.

21. Kang SB, Park JW, Jeong SY, Nam BH, Choi HS, Kim DW, Lim SB, Lee TG, Kim DY, Kim JS, et al: Open versus laparoscopic surgery for mid or low rectal cancer after neoadjuvant chemoradiotherapy (COREAN trial): Short-term outcomes of an open-label randomised controlled trial. Lancet Oncol 11: 637-645, 2010.

22. Bonjer HJ, Deijen CL, Abis GA, Cuesta MA, van der Pas MH, de Lange-de Klerk ES, Lacy AM, Bemelman WA, Andersson J, Angenete E, et al: A randomized trial of laparoscopic versus open surgery for rectal cancer. N Engl J Med 372: 1324-1332, 2015.

23. Jeong SY, Park JW, Nam BH, Kim S, Kang SB, Lim SB, Choi HS, Kim DW, Chang HJ, Kim DY, et al: Open versus laparoscopic surgery for mid-rectal or low-rectal cancer after neoadjuvant chemoradiotherapy (COREAN trial): Survival outcomes of an open-label, non-inferiority, randomised controlled trial. Lancet Oncol 15: 767-774, 2014. 